\title{
Flavor physics in general two Higgs doublet model
}

\author{
Kazuhiro Tobe ${ }^{* \dagger}$ \\ Department of Physics, and Kobayashi-Maskawa Institute for the Origin of Particles and \\ Universe, Nagoya University \\ E-mail: tobe@eken.phys.nagoya-u.ac.jp
}

\begin{abstract}
Motivated by an event excess in Higgs decay $h \rightarrow \mu \tau$ reported by the CMS experiment, we consider a general two Higgs doublet model (2HDM) which predicts the flavor violating phenomena beyond the standard model. Within the framework of the 2HDM, we show the scenario where the $\mu-\tau$ flavor violation can explain the anomaly of the muon anomalous magnetic moment as well as the CMS event excess in $h \rightarrow \mu \tau$. We discuss $\tau \rightarrow \mu \gamma$, the Michel parameters and lepton flavor non-universality in the $\tau$ decays $\tau \rightarrow l v \bar{v}(l=e, \mu)$ as interesting predictions in this scenario.
\end{abstract}

The 3rd International Symposium on "Quest for the Origin of Particles and the Universe" 5-7 January 2017

Nagoya University, Japan

\footnotetext{
* Speaker.

${ }^{\dagger}$ This talk is based on the works in Refs.[1, 2, 3].
} 


\section{Introduction}

Our knowledge of the "flavor" of the elementary particles is still poor. The standard model (SM) of the elementary particle physics is a successful theory, however, we do not have a deep understanding on the "flavor": why we have three generations of quarks and leptons, and how the masses and mixings of quarks and leptons are determined in nature. Therefore, the studies on the flavor related phenomena would be important to obtain the deeper understanding of the flavor and new physics beyond the SM.

Although the SM is very successful, there may be some hints on the flavor as well as physics beyond the SM in the experimental data, which deviate from the SM predictions. For example, the CMS collaboration has reported an event excess in a flavor violating Higgs boson decay $h \rightarrow \mu \tau$ at $\sqrt{s}=8 \mathrm{TeV}$, and the best fit value of the branching ratio is given by

$$
\mathrm{BR}(h \rightarrow \mu \tau)=\left(0.84_{-0.37}^{+0.39}\right) \%,
$$

and the deviation from the SM prediction is $2.4 \sigma$. The ATLAS collaboration has also reported their results $[5,6]$,

$$
\mathrm{BR}(h \rightarrow \mu \tau)=(0.53 \pm 0.51) \%,
$$

which is consistent with the SM prediction as well as the CMS result shown above. Recently the CMS collaboration reported a result based on an integrated luminosity of $2.3 \mathrm{fb}^{-1}$ at $\sqrt{s}=13 \mathrm{TeV}$ and no excess is observed [7]. However it is not sensitive enough to exclude their $8 \mathrm{TeV}$ result, and more data are needed to obtain a conclusive answer to this excess.

Another example of the interesting anomaly is the muon anomalous magnetic moment (muon g-2) as reported by, for example, Ref. [8],

$$
a_{\mu}^{\mathrm{EXP}}-a_{\mu}^{\mathrm{SM}}=(26.1 \pm 8.0) \times 10^{-10}
$$

where $a_{\mu}^{\mathrm{EXP}}\left(a_{\mu}^{\mathrm{SM}}\right)$ is the measured value (the SM prediction) and other groups [9] have also reported the similar results.

One of the simplest extensions of the SM to explain the $\mu-\tau$ flavor violation in the Higgs boson decay is a two Higgs doublet model. Interestingly we have found that the $\mu-\tau$ flavor violation in the 2HDM can explain the muon g-2 anomaly as well as the CMS excess in $h \rightarrow \mu \tau$, simultaneously $[1,2]$. In this talk, I will show this scenario, and then I will discuss the possible predictions of this scenario.

\section{General two Higgs doublet model}

We briefly review a two Higgs doublet model. In a two Higgs doublet model, both neutral components of Higgs doublets get vacuum expectation values (vevs) in general. Taking a certain linear combination, we can always consider a basis (so called Georgi basis or Higgs basis) where only one of the Higgs doublets has the vev as follows:

$$
H_{1}=\left(\begin{array}{c}
G^{+} \\
\frac{v+\phi_{1}+i G}{\sqrt{2}}
\end{array}\right), \quad H_{2}=\left(\begin{array}{c}
H^{+} \\
\frac{\phi_{2}+i A}{\sqrt{2}}
\end{array}\right),
$$


where $G^{+}$and $G$ are Nambu-Goldstone bosons, and $H^{+}$and $A$ are a charged Higgs boson and a CPodd Higgs boson, respectively. We have assumed that the $\mathrm{CP}$ is conserved in the Higgs potential for simplicity. CP-even neutral Higgs bosons $\phi_{1}$ and $\phi_{2}$ can mix and form mass eigenstates, $h$ and $H\left(m_{H}>m_{h}\right)$,

$$
\left(\begin{array}{c}
\phi_{1} \\
\phi_{2}
\end{array}\right)=\left(\begin{array}{cc}
\cos \theta_{\beta \alpha} & \sin \theta_{\beta \alpha} \\
-\sin \theta_{\beta \alpha} & \cos \theta_{\beta \alpha}
\end{array}\right)\left(\begin{array}{c}
H \\
h
\end{array}\right) .
$$

Here $\theta_{\beta \alpha}$ is the mixing angle. Hereafter, we denote $c_{\beta \alpha}=\cos \theta_{\beta \alpha}$ and $s_{\beta \alpha}=\sin \theta_{\beta \alpha}$.

Without imposing an extra symmetry, both Higgs doublets couple to all fermions. In mass eigenbasis for the fermions, the lepton Yukawa interactions are expressed by

$$
\mathscr{L}=-\bar{L}_{L}^{i} H_{1} y_{e}^{i} e_{R}^{i}-\bar{L}_{L}^{i} H_{2} \rho_{e}^{i j} e_{R}^{j}+\text { h.c. },
$$

where $i, j$ represent flavor indices, $L_{L}=\left(V_{\mathrm{MNS}} v_{L}, e_{L}\right)^{T}$, and $V_{\mathrm{MNS}}$ is the Maki-Nakagawa-Sakata (MNS) matrix. Here all fermions $\left(f_{L}, f_{R}\right)(f=e, v)$ are mass eigenstates (i.e. $e_{L, R}^{1}=e_{L, R}, e_{L, R}^{2}=$ $\left.\mu_{L, R}, e_{L, R}^{3}=\tau_{L, R}\right)$. We have assumed the seesaw mechanism with super-heavy right-handed neutrinos to explain the smallness of neutrino masses. The Yukawa coupling matrix $\rho_{e}^{i j}$ is a general $3 \times 3$ complex matrix and can be a source of the Higgs-mediated flavor violating processes. Note that the SM-like Higgs boson $h$ is almost $\phi_{1}$ when $c_{\beta \alpha}$ is close to zero. Therefore, the SM-like Higgs boson $h$ has the flavor violating interactions $\rho_{e}$ via the small mixing $c_{\beta \alpha}$.

From the Higgs potential, one can calculate the mass spectrum of Higgs bosons, and the relations among the Higgs boson masses are expressed as

$$
\begin{aligned}
m_{h}^{2} & \simeq \lambda_{1} v^{2}, \quad m_{H}^{2} \simeq m_{A}^{2}+\lambda_{5} v^{2}, \\
m_{H^{+}}^{2} & =m_{A}^{2}-\frac{\lambda_{4}-\lambda_{5}}{2} v^{2}, \quad m_{A}^{2}=M_{22}^{2}+\frac{\lambda_{3}+\lambda_{4}-\lambda_{5}}{2} v^{2},
\end{aligned}
$$

when $c_{\beta \alpha}$ is small. Here $\lambda_{1,3,4,5}$ and $M_{22}$ are quartic couplings and mass parameter in the Higgs potential, respectively.

\section{3. $h \rightarrow \mu \tau$ and muon g-2 in two Higgs doublet model with $\mu-\tau$ flavor violation}

In this section, we discuss the flavor violating Higgs decay $h \rightarrow \mu \tau$ as well as the muon g-2 in the general 2HDM with $\mu-\tau$ flavor violation $[1,2]$. The expression of the branching ratio for $h \rightarrow \mu \tau$ process is given by

$$
\mathrm{BR}(h \rightarrow \mu \tau)=\frac{\Gamma\left(h \rightarrow \mu^{+} \tau^{-}\right)+\Gamma\left(h \rightarrow \mu^{-} \tau^{+}\right)}{\Gamma_{h}}=\frac{c_{\beta \alpha}^{2}\left(\left|\rho_{e}^{\mu \tau}\right|^{2}+\left|\rho_{e}^{\tau \mu}\right|^{2}\right) m_{h}}{16 \pi \Gamma_{h}},
$$

where $\Gamma_{h}$ is a total decay width of Higgs boson $h$ and here we adopt $\Gamma_{h}=4.1 \mathrm{MeV}$. In order to accommodate the CMS excess, the $\mu-\tau$ flavor violating Yukawa couplings need to satisfy the following condition:

$$
\bar{\rho}^{\mu \tau} \equiv \sqrt{\frac{\left|\rho_{e}^{\mu \tau}\right|^{2}+\left|\rho_{e}^{\tau \mu}\right|^{2}}{2}} \simeq 0.26\left(\frac{0.01}{\left|c_{\beta \alpha}\right|}\right) \sqrt{\frac{\mathrm{BR}(h \rightarrow \mu \tau)}{0.84 \times 10^{-2}}} .
$$


Note that the non-zero $\rho_{e}^{\mu \tau}$ and/or $\rho_{e}^{\tau \mu}$ can explain the CMS excess.

If both $\rho_{e}^{\mu \tau}$ and $\rho_{e}^{\tau \mu}$ couplings are non-zero, the Feynman diagram shown in the left figure of Fig. 1 induces the important contributions to the muon g-2. We note that the chiralities of the muons in external lines have to be flipped in the muon $\mathrm{g}-2$ contributions as shown in the figure. We would
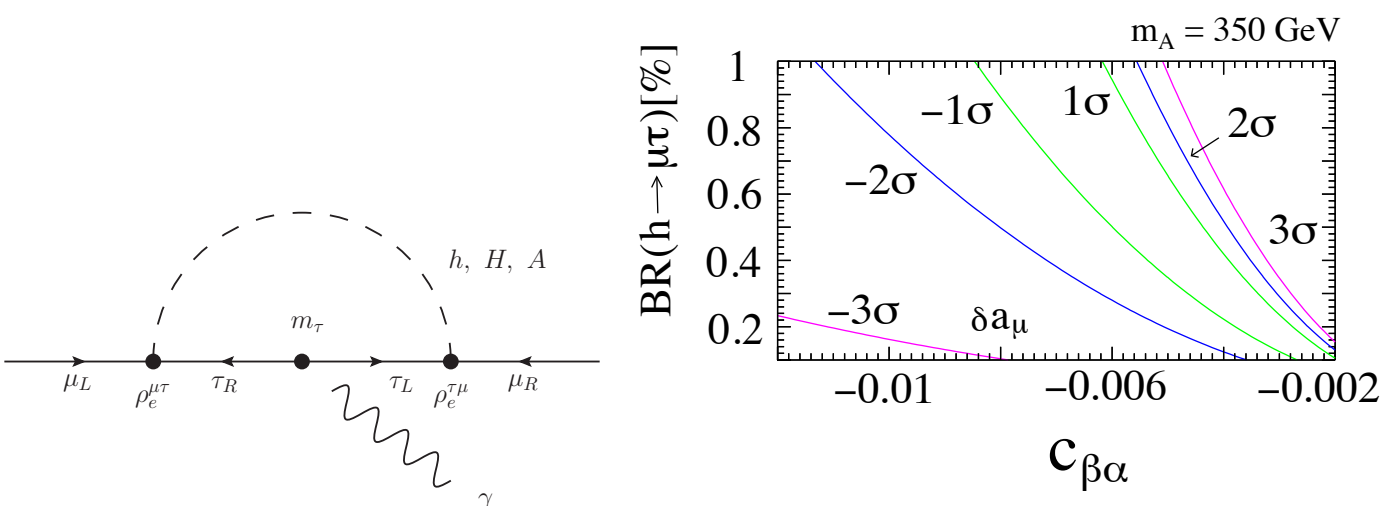

Figure 1: (Left) A Feynman diagram for neutral Higgs boson contributions to the muon g-2 via the $\mu-\tau$ flavor-violating Yukawa couplings $\rho_{e}^{\mu \tau(\tau \mu)}$. A photon is attached somewhere in the charged lepton line. (Right)Neutral Higgs contributions to the muon g-2 $\left(\delta a_{\mu}\right)$ as a function of $\operatorname{BR}(h \rightarrow \mu \tau)[\%]$ and $c_{\beta \alpha}(=$ $\left.\cos \theta_{\beta \alpha}\right)$. The lines where the muon g-2 anomaly is explained within $\pm 1 \sigma, \pm 2 \sigma$ and $\pm 3 \sigma$ are shown. Here we have assumed that $m_{A}=350 \mathrm{GeV}, \lambda_{4}=\lambda_{5}=0.5$ and $\rho_{e}^{\tau \mu}=-\rho_{e}^{\mu \tau}$.

like to stress that normally the chirality flipping is proportional to the muon mass, however, in the presence of both flavor violating couplings $\rho_{e}^{\mu \tau}$ and $\rho_{e}^{\tau \mu}$, the chirality flipping can originate from the mass of the $\tau$, which is in the internal line of the one loop Feynman diagram. Therefore, the $\mu-\tau$ flavor violating Yukawa couplings $\rho_{e}^{\mu \tau(\tau \mu)}$ generate $O\left(m_{\tau} / m_{\mu}\right)$-enhancement in the muon g- 2 contribution. As we discuss above, the Yukawa couplings $\rho_{e}^{\mu \tau(\tau \mu)}$ can be of $O(1)-O(0.1)$ in order to explain the CMS excess in $h \rightarrow \mu \tau$ process, and hence the contributions shown in the left figure of Fig. 1 can be significant. In the right figure of Fig. 1, we show the numerical results for the new contribution to muon $\mathrm{g}-2\left(\delta a_{\mu}\right)$ as a function of $\operatorname{BR}(h \rightarrow \mu \tau)[\%]$ and $c_{\beta \alpha}\left(=\cos \theta_{\beta \alpha}\right)$. The lines where the muon g-2 anomaly is explained within $\pm 1 \sigma, \pm 2 \sigma$ and $\pm 3 \sigma$ are shown. Here we have assumed that $m_{A}=350 \mathrm{GeV}, \lambda_{4}=\lambda_{5}=0.5$ and $\rho_{e}^{\tau \mu}=-\rho_{e}^{\mu \tau}$. As one can see from the figure, there are interesting regions where both the muon g-2 anomaly and the CMS excess in $h \rightarrow \mu \tau$ process are explained if $\left|c_{\beta \alpha}\right|$ are small.

In Refs. [1, 2], we have checked constraints from various processes and concluded that this scenario is still consistent with the current experimental results. In the next section, we will discuss some of the interesting predictions in this scenario.

\section{4. $\tau \rightarrow \mu \gamma[1,2]$}

In the presence of the $\mu-\tau$ flavor violation $\rho_{e}^{\mu \tau(\tau \mu)}, \tau \rightarrow \mu \gamma$ process is generated at one-loop level, similar to the muon g-2, shown in the left figure of Fig. 2. It has been also known that the two-loop contributions to the $\tau \rightarrow \mu \gamma$ process (so-called Barr-Zee type contributions), shown in the right figure of Fig. 2, are significant [10]. 

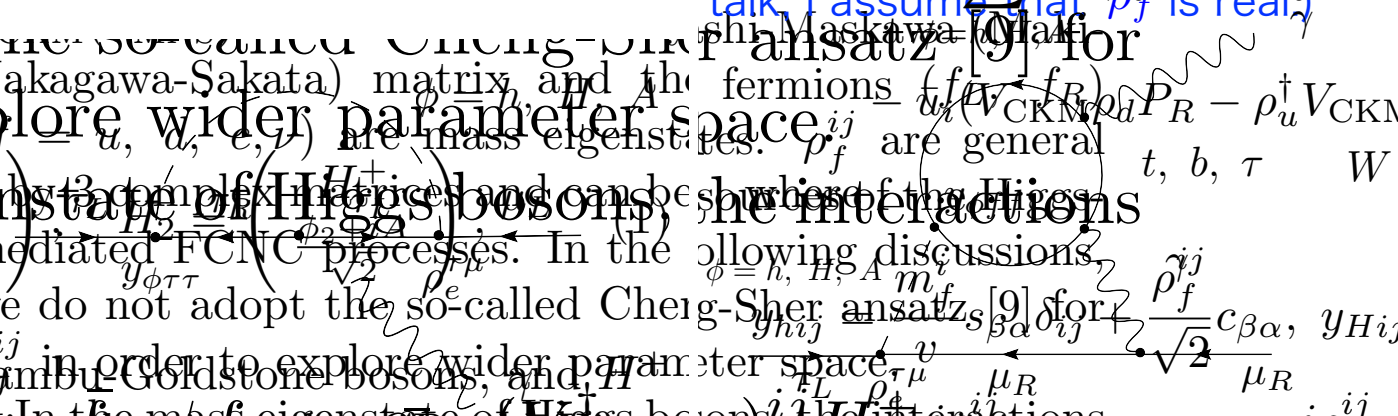

Figure 2: Some of Feynman diagrams which contribute to $\tau \rightarrow \mu \gamma$ process at one-loop level (left figure) and at two-loop level (right figure) which are so-called Barr-Zee type contributions. Diagrams where the fermion chiralities are flipped also contribute to $\tau \rightarrow \mu \gamma$ process.

We note that even if all extra Yukawa couplings $\rho_{f}^{i j}(f=e, u, d)$ except for $\rho_{e}^{\mu \tau(\tau \mu)}$ are negligible, the $\tau \rightarrow \mu \gamma$ process is induced; in this case, the Yukawa couplings $y_{\phi f f}(f=\tau, t, b)$ in Fig. 2 are expressed by the fermion mass $m_{f}$ and the Higgs mixing parameter $c_{\beta \alpha}$ and are not zero, and the predicted branching ratio for $\tau \rightarrow \mu \gamma$ is shown in left figure of Fig. 3, as a function of $\operatorname{BR}(h \rightarrow \mu \tau)[\%]$ and $c_{\beta \alpha}\left(=\cos \theta_{\beta \alpha}\right)$. Here we have assumed the model parameter set same as one in the right figure of Fig. 1. The dependence of $c_{\beta \alpha}$ is very weak once it gets small
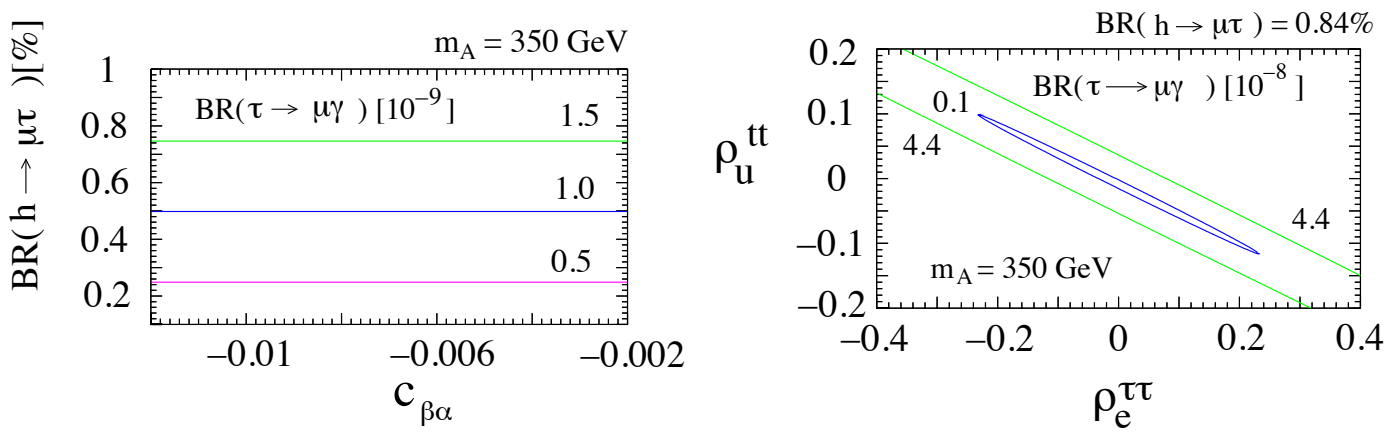

Figure 3: (Left) Branching ratio $\mathrm{BR}(\tau \rightarrow \mu \gamma)$ is shown in case with $\rho_{e}^{\tau \tau}=\rho_{u}^{t t}=0$ as a function of $\operatorname{BR}(h \rightarrow$ $\mu \tau)[\%]$ and $c_{\beta \alpha}\left(=\cos \theta_{\beta \alpha}\right)$. The parameter set of the model is assumed to be the same as one in the right figure of Fig. 1. (Right) Branching ratio in case with non-zero $\rho_{e}^{\tau \tau}$ and $\rho_{u}^{t t}$ as a function of $\rho_{e}^{\tau \tau}$ and $\rho_{u}^{t t}$. Lines for $\operatorname{BR}(\tau \rightarrow \mu \gamma)=4.4 \times 10^{-8}$ (current experimental limit) and $10^{-9}$ are also shown. Here we have assumed the parameter set of the model to be the same as one in the right figure of Fig. 1 except that the $\operatorname{BR}(h \rightarrow \mu \tau)$ and $c_{\beta \alpha}$ are fixed to be $0.84 \%$ and -0.007 and non-zero $\rho_{e}^{\tau \tau}$ and $\rho_{u}^{t t}$ are assumed.

enough. The branching ratio for $\tau \rightarrow \mu \gamma$ gets larger as $\operatorname{BR}(h \rightarrow \tau \mu)$ becomes larger. As can be seen from the figure, the branching ratio is smaller than one in the current experimental limit, $\operatorname{BR}(\tau \rightarrow \mu \gamma)<4.4 \times 10^{-8}$, however, it can be larger than $10^{-9}$, which may be within the reach of the future B-factory.

If other extra Yukawa couplings such as $\rho_{e}^{\tau \tau}$ and $\rho_{u}^{t t}$ are not negligible, they can also generate the extra contributions to the $\tau \rightarrow \mu \gamma$, and hence the branching ratio can be larger. In the right 
figure of Fig. 3, the numerical results for the branching ratio $\mathrm{BR}(\tau \rightarrow \mu \gamma)$ are shown as a function of $\rho_{e}^{\tau \tau}$ and $\rho_{u}^{t t}$. Lines for $\operatorname{BR}(\tau \rightarrow \mu \gamma)=4.4 \times 10^{-8}$ (current experimental limit) and $10^{-9}$ are also shown. Here the parameter set of the model is taken to be the same as one in the left figure of Fig. 3 except that the $\operatorname{BR}(h \rightarrow \mu \tau)$ and $c_{\beta \alpha}$ are fixed to be $0.84 \%$ and -0.007 and non-zero $\rho_{e}^{\tau \tau}$ and $\rho_{u}^{t t}$ are assumed. In this parameter set, the new physics contributions to the muon $\mathrm{g}-2$ is $\delta a_{\mu}=2.2 \times 10^{-9}$, which is within the $1 \sigma$ of the experimental result. As can be seen from the figure, $\rho_{e}^{\tau \tau}$ and $\rho_{u}^{t t}$ can be still of $O(0.1)$ from the current limit. However, the future improvement of the constraint on the branching ratio at the level of below $10^{-9}$ would significantly constrain the parameter space of the scenario.

\section{Michel parameters and lepton flavor non-universality in $\tau \rightarrow l v \bar{v}(l=e, \mu)[2,3]$}

Another interesting prediction of this scenario is the corrections to the $\tau$ decay $\tau \rightarrow \mu v \bar{v}$. As shown in left figure of Fig. 4, the charged Higgs boson contributes to the $\tau$ decay $\tau \rightarrow \mu \nu \bar{v}$ in the presence of non-zero $\rho_{e}^{\mu \tau(\tau \mu)}$. Note that the flavors of neutrinos in the final state are different from those in the SM contributions. Since the neutrinos are not detected experimentally, the charged Higgs contribution induces the corrections to $\tau$ decay $\tau \rightarrow \mu \nu \bar{v}$. Therefore, the detail study of the $\tau$ decay would be interesting to see the new physics effect. For an initial $\tau^{-}$lepton polarization $\mathscr{P}_{\tau}$, the final $\mu^{-}$distribution in the $\tau$ rest frame of $\tau^{-} \rightarrow \mu^{-} v \bar{v}$ decay is given in terms of Michel parameters $\rho_{\mu}, \eta_{\mu}, \xi_{\mu}$ and $\delta_{\mu}[11]$;

$$
\begin{aligned}
\frac{d \Gamma\left(\tau^{-} \rightarrow \mu^{-} v \bar{v}\right)}{d x d \cos \theta_{\mu}} & =\frac{m_{\tau} w^{4}}{2 \pi^{3}} \sqrt{x^{2}-x_{0}^{2}} G_{F_{\mu}}^{2}\left[F_{1}(x)-F_{2}(x) \mathscr{P}_{\tau} \cos \theta_{\mu}\right], \\
F_{1}(x)= & x(1-x)+\frac{2 \rho_{\mu}}{9}\left(4 x^{2}-3 x-x_{0}^{2}\right)+\eta_{\mu} x_{0}(1-x), \\
F_{2}(x) & =\frac{\xi_{\mu} \sqrt{x^{2}-x_{0}^{2}}}{3}\left\{1-x+\frac{2 \delta_{\mu}\left(4 x-4+\sqrt{1-x_{0}^{2}}\right)}{3}\right\},
\end{aligned}
$$

where $G_{F_{\mu}}$ is an effective Fermi constant for $\tau^{-} \rightarrow \mu^{-} v \bar{v}$ process, and $\theta_{\mu}$ is the angle between the $\tau^{-}$spin and the final $\mu^{-}$momentum, $w$ is the maximum $\mu^{-}$energy $\left(w=\frac{m_{\tau}^{2}+m_{\mu}^{2}}{2 m_{\tau}}\right)$, and $x=E_{\mu} / w$ and $x_{0}=m_{\mu} / w$ where $E_{\mu}$ and $m_{\mu}$ are energy and mass for the muon, respectively. Here we have assumed neutrino masses are negligible. The decay rate for $\tau^{-} \rightarrow \mu^{-} v \bar{v}$ is expressed by

$$
\Gamma_{l}=\frac{G_{F_{\mu}}^{2} m_{\tau}^{5}}{192 \pi^{3}}\left\{f\left(y_{\mu}\right)+4 \eta_{\mu} \frac{m_{\mu}}{m_{\tau}} g\left(y_{\mu}\right)\right\},
$$

where $y_{\mu}=\frac{m_{\mu}^{2}}{m_{\tau}^{2}}, f(y)=1-8 y+8 y^{3}-y^{4}-12 y^{2} \log y$, and $g(y)=1+9 y-9 y^{2}-y^{3}+6 y(1+y) \log y$. If only $\rho_{e}^{\mu \tau}$ and $\rho_{e}^{\tau \mu}$ are not negligible in the $2 \mathrm{HDM}$, the Fermi constant and the Michel parameter $\xi_{\mu}$ receive the corrections;

$$
G_{F_{\mu}}=G_{F} \sqrt{1+\Delta^{\mu}}, \quad \Delta \xi_{\mu}=\xi_{\mu}-\xi_{\mathrm{SM}}=-\frac{2 \Delta^{\mu}}{1+\Delta^{\mu}} \simeq-2 \Delta^{\mu}
$$

where $\xi_{\mathrm{SM}}=1$ and $\Delta^{\mu}=\frac{\left|\rho_{e}^{\mu \tau} \rho_{e}^{\tau \mu}\right|^{2}}{32 G_{F}^{2} m_{H^{+}}^{4}}$. Note that in this scenario, $\Delta \xi_{\mu}<0$. In the right figure of Fig. 4, correction to the Michel parameter $\left|\Delta \xi_{\mu}\right|$ is shown as a function of $\operatorname{BR}(h \rightarrow \mu \tau)[\%]$ and 

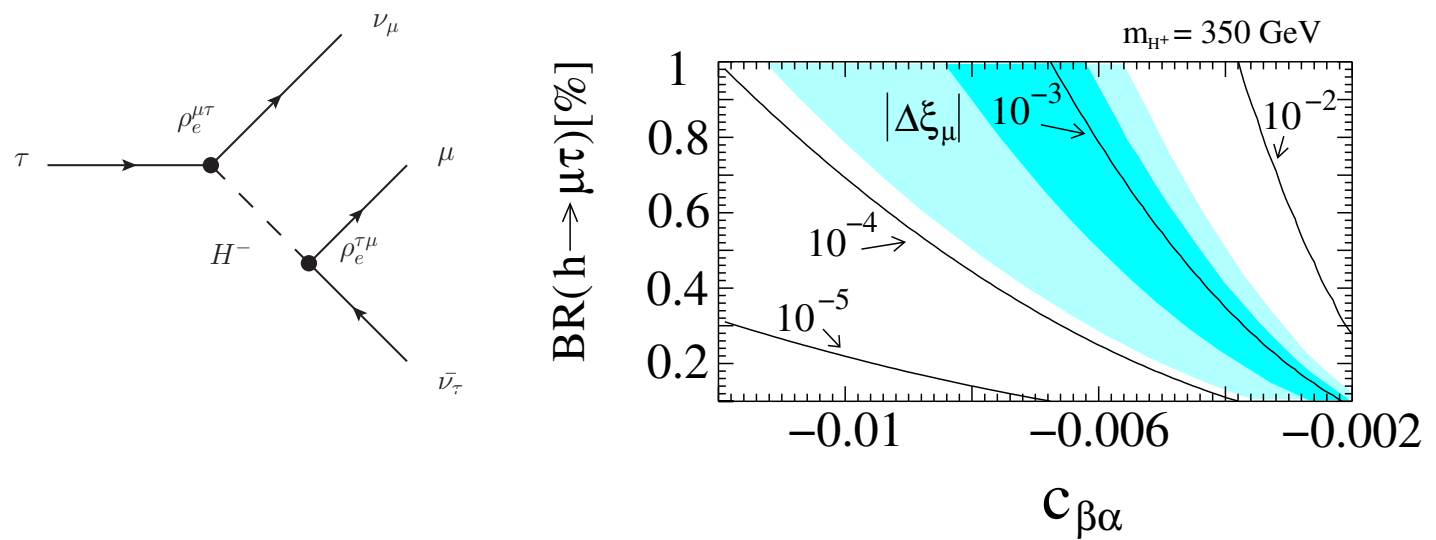

Figure 4: (Left) Feynman diagram for charged Higgs contribution to $\tau \rightarrow \mu \nu \bar{v}$ in the 2HDM with $\mu-\tau$ flavor violation. (Right) Correction to the Michel parameter $\left|\Delta \xi_{\mu}\right|$ as a function of $\operatorname{BR}(h \rightarrow \mu \tau)[\%]$ and $c_{\beta \alpha}\left(=\cos \theta_{\beta \alpha}\right)$. The parameter set of the model is assumed to be the same as one in the right figure of Fig. 1. The dark (light) shaded region can explain the muon g-2 anomaly within $\pm 1 \sigma( \pm 2 \sigma)$.

$c_{\beta \alpha}\left(=\cos \theta_{\beta \alpha}\right)$. The parameter set of the model is assumed to be the same as one in the right figure of Fig. 1. In the figure, the dark (light) shaded region can explain the muon g-2 anomaly within $\pm 1 \sigma( \pm 2 \sigma)$. As can be seen from the figure, interestingly, the corrections between the Michel parameter $\left(\Delta \xi_{\mu}\right)$ and the muon g-2 $\left(\delta a_{\mu}\right)$ are correlated, and hence the precise measurement of $\xi_{\mu}$ at the level of $10^{-4}-10^{-2}$ would probe the interesting region of the parameter space for the muon g-2 anomaly in this scenario. We would like to stress that the precise measurement of the Michel parameters are very important to understand not only the Lorentz and chiral structure but also the flavor structure of the new physics models.

Experiments have also performed a test of lepton flavor universality by measuring the following quantity;

$$
\left(\frac{g_{\mu}}{g_{e}}\right)_{\tau}^{2} \equiv \frac{\operatorname{BR}\left(\tau^{-} \rightarrow \mu^{-} \bar{v} v\right) f\left(y_{e}\right)}{\operatorname{BR}\left(\tau^{-} \rightarrow e^{-} \bar{v} v\right) f\left(y_{\mu}\right)}
$$

where $y_{l}=\frac{m_{l}^{2}}{m_{\tau}^{2}}(l=e, \mu)$ and $f(y)$ is the same function shown in Eq.(5.2). The current world average is $1.0018 \pm 0.0014$ [12]. Interestingly, the correction to the lepton non-universality is sensitive to the lepton flavor violation [2] and it is related to the correction to the Michel parameter $\xi_{\mu}$;

$$
\left(\frac{g_{\mu}}{g_{e}}\right)_{\tau} \simeq 1+\frac{\Delta^{\mu}}{2} \simeq 1-\frac{\Delta \xi_{\mu}}{4}
$$

Since $\Delta \xi_{\mu}<0,\left(g_{\mu} / g_{e}\right)_{\tau}>1$ in this scenario. Therefore the precise measurement of the lepton flavor non-universality at the level of $10^{-4}-10^{-2}$ would be also very interesting to test the solution to the anomaly of the muon $\mathrm{g}-2$. 


\section{Summary}

In this talk, I have discussed the scenario where the $\mu-\tau$ flavor violation in the general 2HDM can explain not only the CMS event excess in $h \rightarrow \mu \tau$ but also the muon g- 2 anomaly, which is still consistent with the various experimental constraints. Needless to say, the more data are needed to get a conclusive answer for the CMS excess in $h \rightarrow \mu \tau$, and certainly they would be very important to understand the theory beyond the SM as well as the mystery of the flavor in the SM.

I have also discussed that one of interesting predictions in this scenario is $\tau \rightarrow \mu \gamma$. The detail predictions depend on the unknown other Yukawa couplings such as $\rho_{e}^{\tau \tau}$ and $\rho_{u}^{t t}$. However, even if the unknown Yukawa couplings except for $\rho_{e}^{\mu \tau(\tau \mu)}$ are negligible, the branching ratio for $\tau \rightarrow \mu \gamma$ can be as large as $10^{-9}$ which would be within the reach of the future B-factory, especially if the value of the branching ratio $\mathrm{BR}(h \rightarrow \mu \tau)$ suggested by the CMS event excess is true. Another prediction discussed here is the Michel parameters and lepton flavor non-universality in $\tau \rightarrow l v \bar{v}(l=e, \mu)$. Because of the $\mu-\tau$ flavor violation, the flavors of neutrinos in the final state are different from those in the SM prediction, and hence the corrections to Michel parameter $\xi_{\mu}$ and lepton flavor violation between $e$ and $\mu$ in $\tau \rightarrow(e, \mu) v \bar{v}$ are induced. Interesting point is that these corrections are correlated with the correction to the muon $\mathrm{g}-2$ without depending very much on the value of $\mathrm{BR}(h \rightarrow \mu \tau)$. Therefore, the precise measurements of these observables would provide the significant test of the solution to the muon $\mathrm{g}-2$ anomaly.

\section{References}

[1] Y. Omura, E. Senaha and K. Tobe, "Lepton-flavor-violating Higgs decay $h \rightarrow \mu \tau$ and muon anomalous magnetic moment in a general two Higgs doublet model," JHEP 1505, 028 (2015) [arXiv:1502.07824 [hep-ph]].

[2] Y. Omura, E. Senaha and K. Tobe, " $\tau$ - and $\mu$-physics in a general two Higgs doublet model with $\mu-\tau$ flavor violation,” Phys. Rev. D 94, no. 5, 055019 (2016) [arXiv:1511.08880 [hep-ph]].

[3] K. Tobe, "Michel parameters for $\tau$ decays $\tau \rightarrow l v \bar{v}(l=e, \mu)$ in a general two Higgs doublet model with $\mu-\tau$ flavor violation," JHEP 1610, 114 (2016) [arXiv:1607.04447 [hep-ph]].

[4] V. Khachatryan et al. [CMS Collaboration], Phys. Lett. B 749, 337 (2015) .

[5] G. Aad et al. [ATLAS Collaboration], JHEP 1511, 211 (2015) .

[6] G. Aad et al. [ATLAS Collaboration], arXiv:1604.07730 [hep-ex].

[7] CMS Collaboration [CMS Collaboration], CMS-PAS-HIG-16-005.

[8] K. Hagiwara, R. Liao, A. D. Martin, D. Nomura and T. Teubner, J. Phys. G 38, 085003 (2011) .

[9] F. Jegerlehner and A. Nyffeler, Phys. Rept. 477, 1 (2009) ; M. Davier, A. Hoecker, B. Malaescu and Z. Zhang, Eur. Phys. J. C 71, 1515 (2011) Erratum: [Eur. Phys. J. C 72, 1874 (2012)] ; F. Jegerlehner and R. Szafron, Eur. Phys. J. C 71, 1632 (2011) .

[10] D. Chang, W. S. Hou and W. Y. Keung, Phys. Rev. D 48, 217 (1993).

[11] L. Michel, Proc. Phys. Soc. A63, 514 (1950); C. Bouchiat and L. Michel, Phys. Rev. 106, 170 (1957); T. Kinoshita and A. Sirlin, Phys. Rev. 107, 593 (1957); T. Kinoshita and A. Sirlin, Phys. Rev. 108, 844 (1957).

[12] B. Aubert et al. [BaBar Collaboration], Phys. Rev. Lett. 105, 051602 (2010) . 